\title{
Deciphering transcription dysregulation in FSH muscular dystrophy
}

\author{
Melanie Ehrlich ${ }^{1}$ and Michelle Lacey ${ }^{2}$
}

DUX4, a homeobox-containing gene present in a tandem array, is implicated in facioscapulohumeral muscular dystrophy (FSHD), a dominant autosomal disease. New findings about DUX4 have raised as many fundamental questions about the molecular pathology of this unique disease as they have answered. This review discusses recent studies addressing the question of whether there is extensive FSHD-related transcription dysregulation in adult-derived myoblasts and myotubes, the precursors for muscle repair. Two models for the role of DUX4 in FSHD are presented. One involves transient pathogenic expression of DUX4 in many cells in the muscle lineage before the myoblast stage resulting in a persistent, disease-related transcription profile ('Majority Rules'), which might be enhanced by subsequent oscillatory expression of DUX4. The other model emphasizes the toxic effects of inappropriate expression of DUX4 in only an extremely small percentage of FSHD myoblasts or myotube nuclei ('Minority Rules'). The currently favored Minority Rules model is not supported by recent studies of transcription dysregulation in FSHD myoblasts and myotubes. It also presents other difficulties, for example, explaining the expression of full-length DUX4 transcripts in FSHD fibroblasts. The Majority Rules model is the simpler explanation of findings about FSHD-associated gene expression and the DUX4-encoded homeodomain-type protein.

Journal of Human Genetics (2012) 57, 477-484; doi:10.1038/jhg.2012.74; published online 21 June 2012

Keywords: expression profiling; FSHD; muscular dystrophy; myogenesis

\section{BACKGROUND ABOUT FSHD}

Facioscapulohumeral muscular dystrophy (FSHD), an autosomal dominant disease, is unique in its linkage to contraction of a tandem repeat array (D4Z4) consisting of large repeat units. ${ }^{1}$ Patients with FSHD1, the predominant form of the disease found in diverse ethnic populations, ${ }^{2,3}$ almost always have a short D4Z4 array in the subtelomeric region of $4 \mathrm{q}(4 \mathrm{q} 35)$. Short arrays contain 1-10 copies of the 3.3-kb repeat unit, instead of $\sim 11-100$. Despite recent insights into the genetics of FSHD, ${ }^{4-6}$ there are still great challenges for understanding the role of dysregulation of gene expression in this disease and the frequent lack of genotype:phenotype correlations. ${ }^{7,8}$

Inside each D4Z4 repeat unit is DUX4, which encodes a transcription factor containing two homeodomains. However, only the most distal copy of DUX4 at 4q35 (Figure 1) is likely to be involved in this painful, debilitating and slowly progressive disease. ${ }^{4-6}$ Diagnosis of FSHD involves molecular analysis of D4Z4 arrays. ${ }^{9-11}$ Usually, the clinical presentation of FSHD is in the second or third decade, with symptoms mostly limited to a small set of skeletal muscles, often exhibiting an asymmetrical distribution of affected muscles. ${ }^{7,12}$ Patients with very small D4Z4 arrays (only one D4Z4 repeat unit) may present in very early childhood. ${ }^{13,14}$ Unlike Duchenne's muscular dystrophy, cardiomyopathy is noted only rarely upon clinical presentation. ${ }^{15}$ Respiratory insufficiency and mild, high-frequency hearing loss are sometimes observed, especially in patients with moderate to severe FSHD. ${ }^{16-19}$ There are frequent associations with asymptomatic retinal telangiectasias. ${ }^{7,12,20}$ No effective treatment is available.

At subtelomeric $10 \mathrm{q}(10 \mathrm{q} 26)$, there is a D4Z4 array that is almost identical to that at $4 \mathrm{q} 35$, with DUX4 in each repeat unit, and that array is similarly polymorphic in size. Much controversy has surrounded the issue of which $4 \mathrm{q} 35$ gene or non-genic DNA sequence is responsible for the linkage of FSHD to contraction of a D4Z4 array at $4 \mathrm{q} 35$ and not at $10 \mathrm{q} 26 .{ }^{21-30} F R G 1$, the gene that is the nearest to $4 q 35$ D4Z4 and absent from 10q26, has been reported to be very strongly upregulated in FSHD muscle; ${ }^{24}$ however, this was not observed in studies from four other labs. ${ }^{25,30-32}$ Genetic evidence indicates that a common single-nucleotide polymorphism (SNP) in the D4Z4-adjacent sequence at the distal end of the array at $4 \mathrm{q} 35$, but not at 10q26, is responsible for the $4 \mathrm{q} 35$ linkage (Figure 1). This SNP, which is present in about half of the general population, ${ }^{33-35}$ confers a

\footnotetext{
${ }^{1}$ Human Genetics Program and Center for Bioinformatics and Genomics, Tulane Cancer Center, Tulane University, New Orleans, LA, USA and ${ }^{2}$ Department of Mathematics, Tulane Cancer Center, Tulane University, New Orleans, LA, USA

Correspondence: Professor M Ehrlich, Human Genetics Program and Center for Bioinformatics and Genomics, Tulane Cancer Center, Tulane University, 1430 Tulane Ave., New Orleans, LA 70122, USA.

E-mail: ehrlich@tulane.edu

or M Lacey, Department of Mathematics, Tulane Cancer Center, Tulane University, 1430 Tulane Ave., New Orleans, LA 70122, USA.

E-mail: mlacey1@tulane.edu
}

Received 30 March 2012; revised 8 May 2012; accepted 21 May 2012; published online 21 June 2012 
Linkage of short D4Z4 arrays and an adjacent distal SNP to FSHD1

FSHD1 : short D4Z4 array \& pA SNP on one 4q35 allele

Short arrays: 1 to 10 D4Z4 3.3-kb units, each containing one DUX4 homeobox gene

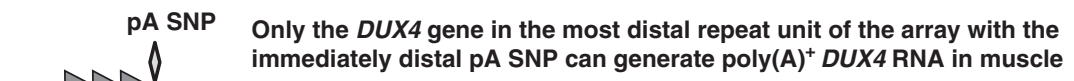

Unaffected : usually long arrays on both allelic $4 q 35$

Long arrays: 11 to $100 \mathrm{D} 4 \mathrm{Z} 4$ units; the vast majority of D4Z4 arrays are long

pA SNP may or may not be present

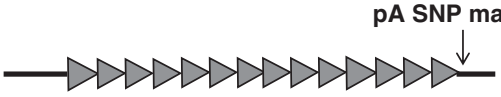

3.3-kb D4Z4 repeat unit containing the 1.1-kb DUX4 gene \& a DUX4 promoter

pA SNP: a SNP conferring a polyadenylation signal missing from the DUX4 gene itself; this pA SNP is present in all FSHD patients \& about $50 \%$ of the general population; rare unaffected individuals have been identified with a short D4Z4 at $4 \mathrm{q} 35$ but no pA SNP

Figure 1 A cartoon illustrating the linkage of short array D4Z4 arrays and an immediately distal SNP to FSHD1.

polyadenylation signal (pA) to the last copy of DUX4 at $4 \mathrm{q} 35$ and is almost never found distal to $10 \mathrm{q}$ D4Z4. ${ }^{4,5}$ No pA is within the DUX4 gene itself, although this gene has a promoter. ${ }^{26}$ The evolutionary conservation of a coding function for DUX4-related sequences ${ }^{36}$ and the absence of any observed FSHD patients with a deletion of the entire D4Z4 array ${ }^{37}$ support FSHD disease models based upon D4Z4 shortening causing inappropriate, gain-of-function expression of DUX4. This gain of function requires the disease-permissive SNP immediately downstream of the last repeat unit in the contracted D4Z4 array. DUX4 is further implicated in FSHD pathogenesis through the discovery of a rare family of FSHD patients with the pA SNP in cis to a short D4Z4 array at 10q26, rather than at $4 \mathrm{q} 35 .{ }^{5}$

Because this misexpressed DUX4 in the FSHD muscle lineage encodes a trans-acting protein, a transcription factor, ${ }^{27}$ any dysregulation of $4 \mathrm{q} 35$ gene expression other than at the D4Z4 array is likely to be a secondary effect comparable to DUX4-initiated FSHD-associated dysregulation of gene expression elsewhere in the genome. This conclusion is re-enforced by the above-mentioned finding of the rare FSHD family with normal-sized 4q35 D4Z4 arrays but with an exceptional pA SNP at 10q26 in cis to a contracted10q26 D4Z4 array. ${ }^{5}$ However, there may well be normal myogenesisassociated changes in chromatin structure at $4 \mathrm{q} 35.2^{38}$ related to the muscle-lineage specific nature of FSHD that could impact the D4Z4 array.

Inappropriate expression of DUX4 to give very low levels of fulllength transcript (DUX4-fl RNA) in FSHD myotubes and myoblasts generally requires both array contraction and the pA SNP. ${ }^{4-6}$ One exception to the requirement for array contraction is the rare variant $\left(\sim 5 \%\right.$ of cases) of FSHD called FSHD2 (or FSHD1B), ${ }^{39,40}$ which needs to be carefully differentiated from other dystrophies that sometimes mimic its clinical symptoms. ${ }^{41}$ Although generation of DUX4-fl RNA in FSHD2 myoblast or myotube nuclei is not linked to contraction of the D4Z4 array, it is still linked to the pA SNP at $4 \mathrm{q} 35 .^{6}$ In addition to DUX4-fl RNA, many shorter transcripts from both strands of D4Z4 are generated in low abundance, ${ }^{42}$ which is not surprising in view of recent findings that a large portion of the human genome is transcribed to generate noncoding RNAs, ${ }^{43}$ especially from DNA repeats. ${ }^{44,45}$ The two DUX4-fl RNA isoforms that have been found associated with FSHD vary only in the $3^{\prime}$ untranslated region, have no identified functional distinction, ${ }^{6,27}$ and will both be referred to as DUX4-fl RNA.

Another exception to the need for array contraction to generate full-length polyadenylated transcripts from DUX4 was seen in multiple normal testis samples, which generate DUX4-fl RNA from both $10 \mathrm{q}$ and $4 \mathrm{q}$ DUX4 at D4Z4. ${ }^{6}$ In testis, the last copy of DUX4 apparently uses either the common pA SNP (at 4q35) or a far distal, constitutively present $\mathrm{pA}$ (at $4 \mathrm{q} 35$ and 10q26) to provide for polyadenylation of the transcript. In addition, an induced pluripotent stem cell culture derived from control fibroblasts was positive for DUX4-fl RNA before, but not after, induction of differentiation. ${ }^{6}$ However, this result awaits confirmation from more samples.

FSHD1, FSHD2 and the germ lineage appear to confer a loose, transcription-conducive conformation to D4Z4 chromatin. This is evidenced by changes in histone modification; ${ }^{46}$ partial but variable hypomethylation of D4Z4 in FSHD1; and more extensive hypomethylation in FSHD2. ${ }^{39}$ There is yet more hypomethylation in normal sperm. ${ }^{47}$ D 4 Z4 hypomethylation does not suffice for the disease as seen in the absence of muscular dystrophy symptoms in patients with ICF (immunodeficiency, centromeric region instability and facial anomlies), the rare, unrelated DNA hypomethylationassociated disease in which D4Z4 is strongly hypomethylated. ${ }^{47,48}$

\section{A MAJOR COMPLICATION IN UNDERSTANDING THE RELATIONSHIP OF DUX4 TO PATHOGENESIS}

DUX4 protein is a transcription factor that can regulate expression of other genes. ${ }^{27}$ Moreover, it contains two homeodomains, ${ }^{49,50}$ domains that are found characteristically in proteins regulating early stages of differentiation, ${ }^{51}$ and localizes to the nucleus. ${ }^{27}$ Therefore, it is easy to envision its inappropriate expression in the muscle lineage leading to pathogenesis. However, a major complication in understanding the relationship of DUX4-fl transcripts to FSHD pathogenesis is their 
extraordinarily low average abundance in FSHD1 and FSHD2 myoblasts, myotubes, and muscle. ${ }^{52}$ Detection of DUX4-fl RNA in FSHD biomaterials requires nested $\mathrm{PCR}^{6}$ or unusually high amounts of cDNA template $(400 \mathrm{ng}){ }^{5,53}$ The need for these non-quantitative conditions for RT-PCR raises fundamental questions about the nature of the causal association of DUX4-fl and the disease pathology. Such PCR conditions also require unusual vigilance to prevent false positives.

It has been estimated that about 1 in 1000 FSHD myoblasts or nuclei in myotubes are positive for DUX4-fl transcripts (by RT-PCR of highly diluted FSHD myoblast cultures) or the corresponding protein in contrast to control cultures, which are negative. ${ }^{6}$ Therefore, the very low levels of FSHD-associated DUX4-fl RNA or protein in cell populations could be accounted for by only a very small percentage of DUX4- $\mathrm{fl}^{+}$nuclei. Moreover, DUX4-fl transcripts were not detectable in some preparations of FSHD myotubes and, especially, FSHD myoblasts. ${ }^{6,53}$

\section{TWO MODELS TO EXPLAIN DUX4-FL PATHOGENICITY}

At clinical presentation, affected muscles in FSHD patients have heterogeneous and rather non-specific histological findings. ${ }^{13,54}$ Although Reed et al. ${ }^{55}$ found a significant increase in the distance between the sarcolemma and the underlying contractile apparatus in unfixed FSHD skeletal muscle, the basic contractile apparatus was normal by immunofluorescence and confocal microscopically. We will consider models of pathogenesis focused on abnormal regenerative repair of muscle ${ }^{56}$ in FSHD. This focus is supported by the typically slow progress of the disease and the usual presentation of symptoms after the first decade of life.

Satellite cells, which account for only $\sim 2-6 \%$ of the nuclei in adult skeletal muscle, are the main source of stem cells for repair of postnatal skeletal muscle through regenerative myogenesis, induced by muscle wear-and-tear, injury, or disease-related atrophy. ${ }^{56-58}$ During regenerative myogenesis, satellite cells are induced to proliferate and form myoblasts. These mononuclear cells then differentiate and fuse with the damaged myofiber or with other myoblasts to form multinucleated myotubes. A key question for understanding the biological implications of the very infrequent DUX4- $\mathrm{fl}^{+}$nuclei (at the RNA or protein level) in FSHD myoblast and myotube cultures derived from affected muscle is whether a large fraction of these cells has a disease-associated expression phenotype.

We consider two models inferred from recent articles about FSHD. ${ }^{4-6,53,59-61}$ Model 1 ('Minority Rules') involves undefined molecules spreading toxicity initiated by DUX4-fl protein in the tiny percentage of FSHD myoblasts or nuclei in FSHD myotubes that are positive for DUX4-fl. In this model, there is no need for a diseaselinked expression profile in the vast majority of the cells in the population (Figure 2). Currently, there is an emphasis in the literature on the toxic and pro-apoptotic effects of DUX4 as central to pathogenesis, as deduced from experiments involving the introduction of plasmid or viral DNA constructs encoding moderate to high amounts of DUX4-fl RNA in various cell types. ${ }^{4,6,59,62}$ Even at low, non-toxic concentrations of experimentally induced DUX4-fl RNA in mouse myoblasts, there is downregulation of Myod1 RNA levels and concomitant inhibition of myotube formation and expression. ${ }^{59}$ When myoblasts are undergoing differentiation to myotubes, DUX4-fl constructs are less toxic but, nonetheless, upregulate atrophy-associated genes (FOXO32/MURF1 and TRIM63/ ATROGIN-1) and lead to decreased yields of myotubes or myotubes of very abnormal morphology upon differentiation of myoblasts. ${ }^{59,63}$
In contrast to Model 1, Model 2 ('Majority Rules') is predicated on a large fraction of FSHD myoblasts (and myotube nuclei) exhibiting a disease-associated expression phenotype in the absence of detectable DUX4- $f l$ RNA in $>99 \%$ of the nuclei (Figure 2). The diseaseassociated expression phenotype would include genes whose abnormal up- or downregulation leads to defects in muscle regeneration and function. Model 2 explains the discrepancy between DUX4-fl protein expression in only a tiny fraction of FSHD myoblasts and a large percentage of these cells having disease-linked alterations in expression as a result of transient, non-toxic expression of DUX4-fl RNA in a large fraction of the cells previous to the myoblast stage. This short-term inappropriate expression of DUX4 would initiate an irreversible cascade of gene dysregulation, similar to the way that other homeobox genes direct gene expression profiles by early transient expression during differentiation. ${ }^{51}$ The expression of DUX4-fl RNA at the myoblast (and myotube or later) stages in a tiny fraction of nuclei in FSHD cells would be a stochastic event that is peripheral to the establishment of pathogenesis. Moreover, DUX4-fl expression in the very small fraction of myoblast or myotube nuclei expressing the gene might be toxic but it would not be toxic at the pre-myoblast stage, according to this model.

A variant of Model 2 (not shown) would involve oscillating, non-toxic generation of DUX4-fl transcripts throughout the FSHD myotube population such that, at any one time, $<1 \%$ of the nuclei are generating the transcript. Such oscillating expression, which is seen for genes encoding certain transcription regulatory factors in stem cells, ${ }^{64-66}$ might reinforce an FSHD expression phenotype set up at a pre-myoblast stage and might avoid inhibition of myotube formation if the expression were very transient to give much lower intracellular levels of DUX4-fl protein than in transfection and transduction experiments. ${ }^{4,59,63}$ DUX4-fl constructs have been reported to be less toxic at the myotube stage. ${ }^{59,63}$ However, strong induction of expression of DUX4-fl in differentiating or differentiated myoblasts transduced or transfected with DNA constructs still resulted in some cell death, gave very abnormallooking myotubes, and increased levels of expression of genes associated with muscle atrophy. ${ }^{59,63}$

In the above-described models, stochastic effects determining the generation of DUX4-fl RNA and protein could contribute to the lack of consistent phenotype-genotype correlations, the asymmetry in affected skeletal muscle upon clinical presentation, and the finding that FSHD symptoms are usually not seen earlier than the second decade. ${ }^{7,8}$ Moreover, there may be more of a range of expression levels of DUX4-fl RNA and protein per FSHD myogenic cell or nucleus than is currently appreciated. In addition, the levels or timing of inappropriate expression of DUX4-fl in vivo in myogenic precursors (or the extent of dysregulation of downstream genes) may be different than in vitro, just as is the case for the reprogramming factor Zcan4 in embryos vs embryonal stem cells ${ }^{67,68}$ (see below). Nonetheless, FSHD muscle appears to have much lower levels of DUX4-fl expression than cultured FSHD myotubes and is sometimes undetectable in affected FSHD muscle biopsies, ${ }^{42,52,61}$ a finding that is consistent with the models based upon the central role of myogenic precursor cells in disease pathogenesis.

\section{EVIDENCE FOR A DISEASE-ASSOCIATED TRANSCRIPTION PHENOTYPE IN FSHD MYOBLASTS AND MYOTUBES: SUPPORT FOR THE 'MAJORITY RULES' MODEL}

Expression microarray profiling of FSHD vs control muscle biopsy samples has been done, but with little consistency in the results. ${ }^{31,32,69-71}$ This is probably due partly to the use of very 

molecules necessary for pathogenesis through toxic effects and inhibition of regenerative myogenesis.

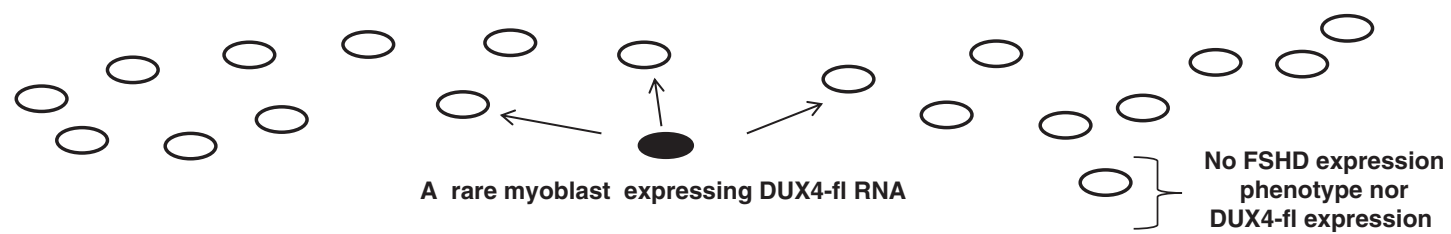

Model 2 (Majority Rules) : Transient expression of DUX4-fl at a pre-myoblast stage changes the expression profile in most cells

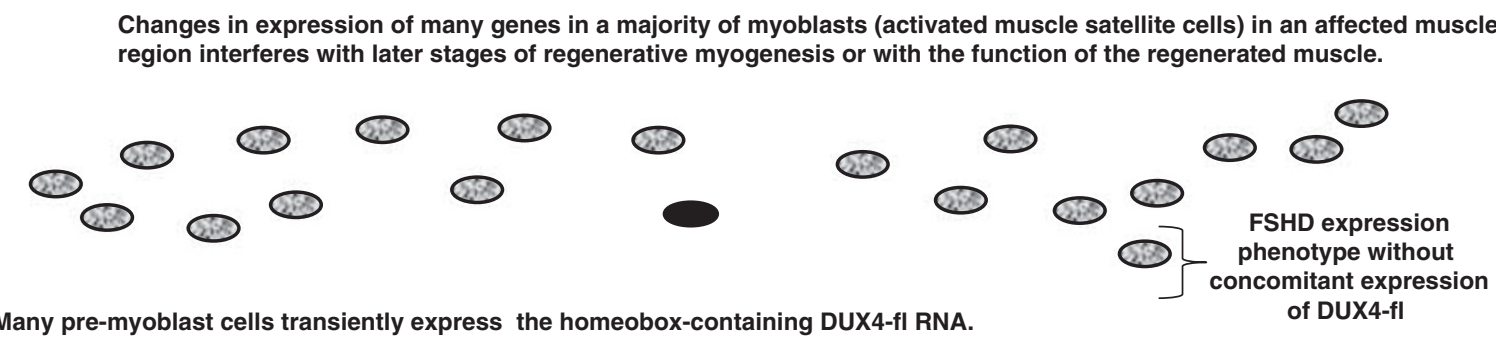

Rare re-expression in myoblasts or myotubes is seen but is not necessary for initiating pathogenesis.

Figure 2 Two models for inappropriate DUX4 expression initiating FSHD pathogenesis. Both the Minority Rules model (Model 1) and the Majority Rules model (Model 2) involve only certain skeletal muscles being affected and generally slow progression with age. A variant of Model 2 would have transient oscillating expression of DUX4-fl at the myotube stage reinforcing an FSHD expression phenotype established at a pre-myoblast stage.

different types of microarrays, none of which were the recently improved, exon-based microarrays. Moreover, studies of muscle are complicated by various extents of contamination with non-muscle cells. In addition, myogenesis-specific changes in muscle tissue would be obscured by the very low percentage of satellite cells in muscle tissue. Recently, four multi-gene expression studies were reported for FSHD and control myoblasts and myotubes that used either exonbased microarrays ${ }^{53,72}$ or panels of genes for qRT-PCR. ${ }^{60,61}$ Although different experimental methods or types of samples were used, which probably contribute to considerable differences between the gene expression profiles, these studies present evidence for disease-linked dysregulation of many genes in FSHD myoblasts and myotubes.

First, we summarize results from our expression profiling of immunocytochemically characterized myoblast and myotube preparations $^{53}$ using myoblast cultures that were $\sim 70 \%$ confluent or that had been in differentiation medium for $4-6$ days. Of the $\sim 17000$ analyzed genes on the exon-based microarray, 295 and 797 were significantly dysregulated in FSHD vs control myoblasts and myotubes, respectively (fold change $>2$; adjusted $P<0.01$ ). Many genes that displayed disease-related dysregulation (for example, genes for muscle structure, mitochondrial function and signal transduction) exhibited a dampening, but importantly, not the absence of normal myogenesis-specific expression changes. Some critical myogenesisassociated genes (for example, MYOD1 and MYOG) displayed normal levels of expression in FSHD myoblasts and myotubes, consistent with the normal growth and differentiation of the FSHD myoblasts and normal appearance of FSHD myotubes. However, other regulatory genes (for example, $M E F 2 A$ and all four of the Argonaute genes) that could have widespread effects on gene expression were dysregulated in FSHD myogenic cells. ${ }^{53}$ That 60 genes showed about 4-16-fold downregulated RNA levels in FSHD vs control myotube preparations indicates a high percentage of the nuclei displaying FSHD-associated dysregulation of transcription.

Our findings suggest that FSHD-related changes in gene expression contribute to abnormalities in muscle function and structure in FSHD. Among the most overrepresented functional terms associated with upregulated genes in FSHD vs control myotubes were inflammation, fatty acid elongation in mitochondria and extracellular matrix. ${ }^{53}$ These could be relevant to the inflammation, fatty acid infiltration or fibrosis that has been observed in a varying percentages of FSHD muscle biopsies. ${ }^{18,73-76}$ For example, expression of the proinflammatory genes IL6, IL8, IL18R1, BDKRB1, CCL2, CCL20, TNFAIP6 and TNFRSF12A was upregulated in FSHD myotubes (fold change $>2$; adjusted $P<0.01$ ). The fibrosis-associated CTGF, which was found to be upregulated at the RNA level in FSHD vs control muscle ${ }^{32}$ and at the RNA and protein level in muscle fibers from patients with other muscular dystrophies, ${ }^{77}$ was upregulated threefold in FSHD vs control myotubes. ${ }^{53}$

Secondly, in an expression profiling study similar to ours, Cheli et al. ${ }^{72}$ concluded that there was specific dysregulation in myoblasts and myotubes from FSHD patients vs. controls. However, their data are unconvincing because of the lack of assessment of the quality of their myoblast and myotube preparations by immunocytochemistry. Indeed, paradoxically, they reported no muscle-related terms among 177 functional terms associated with genes differentially expressed in control myoblast vs control myotube preparations. In contrast, as expected, in our microarray study all six of the top functional terms for genes displaying differential expression in control myoblasts vs control myotubes had the term 'muscle' in them. ${ }^{53}$ In addition, Cheli et al. reported $<4 \%$ overlap between several hundred genes with dysregulation in FSHD vs control cells at the myoblast stage and those dysregulated at the myotube stage, unlike in our study in which there 
was $48 \%$ overlap between myoblasts and myotubes for the genes with FSHD dysregulation (fold-change $>2 ; P<0.01$ ).

Thirdly, Homma et al. ${ }^{60}$ analyzed by qRT-PCR the relative expression of 64 test genes and 11 control genes in wellcharacterized FSHD and control myoblasts at five time points before or after induction of differentiation to myotubes. ${ }^{60}$ Their subjects were cohorts of affected and unaffected family members, with myoblasts generated from the deltoid and bicep biopsies of each subject. A hierarchical clustering analysis demonstrated that there were strong cohort-related groupings among the expression profiles and that most of the correlations in expression profiles between samples from closely related patients were stronger than the correlations between pairs of FSHD samples or pairs of control samples. The authors then analyzed the average differences in expression between the FSHD and control samples at each time point using standard $t$-tests and concluded that there were 'no consistent, overall differences in mRNA expression patterns or levels' between FSHD and control myoblasts. However, this analysis was statistically flawed in two critical respects: (1) the measurements at each time point were treated as independent rather than as repeated measures from each subject and (2) there was no adjustment for the variation associated with the sample cohorts. To remedy these deficiencies and thereby greatly increase the power to detect statistically significant FSHD-related differences, we fit mixedeffects models to predict the PCR-derived $\mathrm{Ct}$ value as a function of time and/or sample type for their data from the last three differentiation time points $(2,4$ and 7 days in differentiation medium). We used nested random intercepts to account for varying baseline levels among and within each cohort, and modeled the effect of FSHD status as an additive term and, where statistically significant, as an adjustment to the time-related slope coefficient. By this re-analysis, we identified 13 genes with significant FSHD-related dysregulation of expression from their data (CXCL11, KLF4 and FRG2B, upregulated; ACTN3, DES, MYH5, MYH6, MYH14, PGK1, SULF2, FBXO32/ATROGIN1, TRIM63/MURF1 and SLC25A4/ANT1, downregulated). The SLC25A4 downregulation is contrary to previous findings, ${ }^{24,30,78}$ and the specificity of $F R G 2 B$ probes remains to be demonstrated for this gene, which has very similar sequences throughout the genome. In our expression profiling, six of the 64 genes common to the study of Homma et al. ${ }^{60}$ and ours ${ }^{53}$ displayed significant FSHD-related downregulation of at least twofold (DES, MYF6, MYH6, MYH7, TRIM63/MURF1). Three of these were also significantly downregulated in our re-evaluation of the data of Homma et al. (DES, MYH6 and TRIM63). Of the 64 genes, 50, including FSHD candidate gene PITX1, ${ }^{24,27}$ displayed no significant differential expression in either study. The $P$-value for the relationship between the direction of differential expression between the two studies was 0.09 (Fisher's exact test), providing some evidence of an association, although not at a statistically significant level.

Given the lack of normalization of the qRT-PCR data from test genes to standard genes by Homma et al. and their unconventional use of a pre-amplification for 14 cycles before the real-time PCR, the finding of significant downregulation for three genes in both of these studies is noteworthy. Moreover, all three of these genes are strongly upregulated in control myotubes vs 19 different non-muscle cell type, ${ }^{53}$ and were seen as upregulated in myoblasts vs non-myogenic cells in RNA-seq (http://genome.ucsc.edu/ENCODE/, Tom Gingeras, Cold Spring Harbor). This is consistent with our finding that one of the most prominent classes of genes to be dysregulated in FSHD myotubes was genes normally upregulated during myogenesis. In summary, the conclusion of Homma et al. that FSHD and control myogenic precursors have indistinguishable patterns of gene expression is not supported by our re-analysis of their data.

In the last of these recent expression studies, Geng et al. ${ }^{61}$ transduced human myoblast cultures with DUX4-fl or control constructs and profiled differential gene expression with an expression microarray $24 \mathrm{~h}$ after transduction. The short time of incubation was probably intended to minimize the contribution of toxic effects of induced DUX4- $f l$ expression to myoblasts, although it is likely that such effects still altered expression of many genes. Among the more than 1000 genes that were significantly dysregulated (fold change $>2$; false discovery rate $<0.01$ ) by the DUX4-fl construct was a small group of genes strongly upregulated due to transduction with DUX4-fl and expressed specifically in germ cells or during early development. By qRT-PCR, Geng et al. showed that six of these genes (ZSCAN4, KHDC1, PRAMEF1, RFPL2, MBD3L2 and TRIM43) were expressed at moderate levels in normal testis and confluent FSHD myoblasts. The steady-state levels of their RNAs were usually much lower in FSHD skeletal muscle samples, and they displayed little or no expression in control muscle or confluent control myoblasts. These results are consistent with roles for these genes in gametogenesis and abnormal regenerative myogenesis in FSHD.

The only one of these six genes with a known function is ZSCAN4/ Zscan4, which is expressed specifically at the late two-cell stage and promotes the normal progression of mouse embryos to the four-cell stage. ${ }^{67}$ The transient expression of this gene regulates pluripotency, genome stability, and telomere stability and can upregulate expression of several hundred genes during the late stages of induced pluripotent stem cell formation with major changes in phenotypic outcome. ${ }^{79} \mathrm{We}$ noticed that among the genes regulated by Zscan 4 are the murine homologs of KHDC1, TRIM43 and PRAMEF7, ${ }^{79}$ all of which were found to be upregulated by DUX4-fl transduction. ${ }^{61}$ The first two of these genes were also analyzed in human samples and shown to be testis- and FSHD-associated. ${ }^{61}$ The $1.9-\mathrm{kb}$ enhancer and promoter region of ZSCAN4 contains four binding sites for DUX4-fl protein and was responsive to strong upregulation by transduced DUX-fl in a reporter gene assay using a human rhabdosarcoma cell line. ${ }^{61}$ Given its ability to upregulate many genes during early differentiation, this gene might be one of the earliest to be dysregulated by inappropriate expression of DUX4-fl in the FSHD muscle lineage and could have a major role in establishing the FSHD transcription phenotype.

Four of the above six testis/FSHD muscle-lineage genes, ZSCAN4, $P R A M E F 1, K H D C 1$ and RFPL2, were included in our expression array study. ${ }^{53}$ We found that ZSCAN4, PRAMEF1 and KHDC1 were upregulated $\sim 4$-, 5- and 2-fold (adjusted $P=3 \times 10^{-6}, 10^{-4}$ and $10^{-4}$ ), respectively, in FSHD vs control myotubes with only about 1.5-fold upregulation in FSHD cells at the myoblast stage. Some of the hundreds of changes in gene expression from myoblasts to myotubes $^{53}$ may be responsible for our observing a stronger FSHDassociated upregulation of levels of these transcripts at the myotube stage. We used myoblasts from $70 \%$ confluent cultures, which are not committed to myotube formation, unlike Geng et al. ${ }^{61}$ who used confluent myoblast cultures, and this may account for their higher FSHD-specific upregulation of these genes at the myoblast stage. The testis association of these genes probably reflects the finding that the only normal postnatal tissue shown to express DUX4-fl RNA and protein is testis. ${ }^{6}$ Another testis-associated gene, CCNA1, which encodes a meiosis-associated cyclin, was strongly upregulated in control myoblasts transduced with a DUX4-fl expression construct in the study of Geng et al. ${ }^{61}$ Analogously, we found strong upregulation of this gene, 3.6- and 24-fold, in FSHD vs control myoblasts and myotubes, respectively (adjusted $P<0.01$ and $\left.10^{-6}\right){ }^{53}$ 
In summary, several studies indicate that multiple genes are dysregulated in normal-appearing FSHD myoblasts and myotubes.

\section{OTHER EVIDENCE FAVORS THE 'MAJORITY RULES' MODEL}

Because DUX4 is implicated in FSHD and is a homeobox gene, it is important to consider the nature of homeobox genes in evaluating models for DUX4 pathogenicity. The expression of homeobox genes is tightly regulated temporally as well as being highly specific for cell type, in accord with their ability to select developmental fates. ${ }^{51,80-82}$ Although some homeobox genes are expressed in specific adult cell populations in which they have maintenance or cell-survival functions, generally, expression of this class of genes in higher eukaryotes is most prominent in the early stages of cellular differentiation. ${ }^{82-86}$ Expression of many homeobox genes depends on their long-range chromatin epigenetic environment and is facilitated by their frequent clustering. ${ }^{82-84,87}$ Their expression is also a function of the presence of other early differentiation-associated proteins $^{82,83}$ as well as post-transcriptional and post-translational control. ${ }^{88,89}$

Model 2 (Majority Rules, Figure 2) involves DUX4-fl protein, at certain developmental stages, not inducing acute toxicity, but rather causing aberrant modulation of the transcription program. A parallel to this hypothesis is the finding that even typical homeobox genes like HOXA5 and PITX1 can cause p53-mediated apoptosis upon aberrant upregulation in certain cell types. ${ }^{90,91}$ Specific epigenetic and transcription factor determinants of expression of homeobox genes and their association with early stages of development could explain why DUX4-fl RNA might be generated transiently from a high percentage of FSHD muscle precursor cells only at a pre-myoblast, probably satellite-cell stage. Similarly, the biological activity of the homeodomain proteins encoded by homeobox genes is highly dependent on cell type. This includes the need for stage-specific DNA-binding partners and proteins that affect the activity of homeodomain proteins once they are bound to their DNA target. ${ }^{92,93}$

Evidence that DUX4- $\mathrm{fl}$ is not toxic at certain cell stages is seen in FSHD fibroblast-derived induced pluripotent stem cell cultures. ${ }^{6}$ These differentiate to give embryoid bodies of normal appearance and cell composition, despite the evidence for a higher abundance of DUX4-fl RNA in these embryoid bodies than in FSHD myoblasts. ${ }^{6}$ Moreover, the finding of long DUX4-fl RNA and corresponding protein encoded at both $4 \mathrm{q} 35$ and $10 \mathrm{q} 26$ DUX4 in normal testis ${ }^{6}$ suggests a genetically programmed function for D4Z4-derived DUX4 protein at some stage(s) in normal human development that is yet to be determined.

In addition to the tandem arrays at $4 \mathrm{q} 35$ and $10 \mathrm{q} 26$, representatives of paralogous groups of mammalian DUX-type double-homeobox sequences are present in many locations in the human genome. ${ }^{94}$ The only DUX-related gene with evidence as to its specific function is mouse Duxbl, whose expression pattern implicates it in gametogenesis, thymocyte maturation and prenatal myogenesis in mice. ${ }^{95,96}$ Knockout of this gene confirmed its role in the production of murine $\mathrm{CD}^{+} / \mathrm{CD}^{+}{ }^{+}$thymocytes. ${ }^{96}$ However, Leidenroth and Hewitt ${ }^{36}$ concluded that Duxbl is not an otholog of DUX4. Therefore, it is unclear whether functions of Duxbl are relevant to DUX4. Moreover, they found that evolutionary descent of DUX4 from a $D U X C$-type precursor is more likely than from a DUXB precursor. $^{36}$ They described a tandem repeat of the DUXC-related rodent Dux gene as the most likely functional equivalent of DUX4, and it is of unknown function.

With respect to models that propose that the toxicity of DUX4 expression in myoblasts or myotubes is central to pathogenesis
(Minority Rules, Model 1), C2C12 cells transduced with PAX3 or $P A X 7$ constructs plus a DUX4 construct can be spared the acute toxicity from DUX4-fl expression. ${ }^{59} P A X 3$ and $P A X 7$ have important roles in embryonic and early postnatal myogenesis, and $P A X 7$ RNA is persistently found in adult satellite cells. ${ }^{86}$ Because PAX7 RNA is also present in undifferentiated myoblasts, which are nonetheless susceptible to DUX4-fl toxicity, the relevance of PAX7 to DUX4 and FSHD pathogenicity is unclear. The sequence similarity of the homeodomains of PAX7 and DUX4 have been invoked in hypotheses about PAX7 and DUX4 competing for DNA binding. ${ }^{59}$ However, other homeodomain proteins in addition to PAX7 have similar homeodomains to those of DUX4 and so also may compete with DUX4-fl for binding to DNA. ${ }^{36}$ In any event, PAX3 and PAX7 offer paradigms for how the spatio-temporally limited presence of transcription factors can counteract DUX4-fl toxicity.

The hallmarks of DUX4-fl toxicity in myoblasts are the loss of cell viability, MYOD1/Myod1 downregulation, inhibition of myotube formation or formation of very abnormal-looking myotubes, and upregulation of atrophy-associated FBXO32/ATROGIN1and TRIM63/ MURF $1 .{ }^{59,63}$ None of these changes were observed by us or by Homma et al. ${ }^{53,60}$ upon examination of many FSHD myoblast cell strains; see above for evidence of FSHD downregulation, rather than upregulation, of TRIM63. Both of these groups and that of Barro et al. ${ }^{53,60,97}$ found that FSHD and control myoblasts cannot be distinguished by viability, growth rates or rates of differentiation to myotubes. Although some differences in the shape of FSHD and control myotubes were reported, ${ }^{63,97}$ we and Homma et al. found the shape of myotubes to be variable among both control and FSHD cell strains with no disease association..$^{53,60}$ The good growth and differentiation of FSHD myoblasts should reflect the population of satellite cells in vivo because FSHD myoblast cell strains from moderately affected muscle are no more difficult to generate than control myoblast cell strains and can undergo similar numbers of cell population doublings. ${ }^{53}$ However, it is possible that FSHD myoblasts from severely affected muscle, which are difficult to propagate, have more frequent expression of DUX4-fl RNA and protein.

Another finding favoring Model 2 over Model 1 is that FSHD fibroblasts contain DUX4-fl transcripts at very low levels, as do FSHD (but not control) myoblasts, myotubes and muscle tissue. ${ }^{6}$ FSHD is predominantly a skeletal muscle-specific disease. Therefore, it is likely that the inappropriate DUX4 expression that establishes pathogenesis is mostly specific to the muscle lineage and would not be shared with fibroblasts. Unlike Model 1, Model 2 with its postulated frequent expression of DUX4-fl at a muscle lineage-specific pre-myoblast stage obviates the difficulty posed by FSHD fibroblasts expressing DUX4-fl RNA.

\section{CONCLUSIONS}

A large fraction of FSHD myoblasts and myotubes from moderately affected muscle displays an expression dysregulation phenotype. This and other findings support a model invoking transient, pathogenic expression of DUX4 in a large fraction of cells at the pre-myoblast stage (Figure 2, Model 2). Among the dysregulated targets of the hypothesized burst of DUX4 expression in this model could be the genes encoding the testis-and very early embryogenesis-specific reprogramming and telomere-stabilizing factor ZSCAN4 and the meiosis-associated cyclin CCNA1. ${ }^{79,98}$ These genes were upregulated by transduction of DUX4 constructs into control myoblasts. ${ }^{61}$ In FSHD patients, we propose that there is transient expression of DUX4-fl at a pre-myoblast stage in affected regions of skeletal muscle and possibly among certain subsets of muscle satellite cells. ${ }^{99,100}$ This 
could result in upregulation of expression of these and other genes and, during regenerative myogenesis, the dampening of expression of many muscle lineage-associated genes. ${ }^{53}$ This, in turn, could decrease the efficiency of the late stages of regenerative myogenesis or affect muscle function in a manner consistent with the usually slow progression of FSHD.

\section{ACKNOWLEDGEMENTS}

This article is dedicated to the memory of Carol A Perez, who helped to found the FSH Society, and worked with uncommon devotion and intelligence on behalf of FSHD patients. Supported in part by the FSHD Global Research Foundation and NIH Grant NS048859.

1 Hewitt, J. E., Lyle, R., Clark, L. N., Valleley, E. M., Wright, T. J., Wijmenga, C. et al. Analysis of the tandem repeat locus D4Z4 associated with facioscapulohumeral muscular dystrophy. Hum. Mol. Genet. 3, 1287-1295 (1994).

2 Matsumura, T., Goto, K., Yamanaka, G., Lee, J., Zhang, C., Hayashi, Y. K. et al. Chromosome 4q;10q translocations; comparison with different ethnic populations and FSHD patients. BMC Neurol. 2, 7 (2002).

3 Lemmers, R. J., van der Vliet, P. J., van der Gaag, K. J., Zuniga, S., Frants, R. R., de Knijff, P. et al. Worldwide population analysis of the $4 q$ and $10 q$ subtelomeres Identifies only four discrete interchromosomal sequence transfers in human evolution. Am. J. Hum. Genet. 86, 364-377 (2010).

4 Kowaljow, V., Marcowycz, A., Ansseau, E., Conde, C. B., Sauvage, S., Matteotti, C. et al. The DUX4 gene at the FSHD1A locus encodes a pro-apoptotic protein. Neuromuscul. Disord. 17, 611-623 (2007).

5 Lemmers, R. J., van der Vliet, P. J., Klooster, R., Sacconi, S., Camano, P., Dauwerse, J. G. et al. A unifying genetic model for facioscapulohumeral muscular dystrophy. Science 329, 1650-1653 (2010).

6 Snider, L., Geng, L. N., Lemmers, R. J., Kyba, M., Ware, C. B., Nelson, A. M. et al. Facioscapulohumeral dystrophy: incomplete suppression of a retrotransposed gene. PLoS Genet. 6, e1001181 (2010).

7 Tawil, R. Facioscapulohumeral muscular dystrophy. Neurotherapeutics 5, 601-606 (2008).

8 Scionti, I., Fabbri, G., Fiorillo, C., Ricci, G., Greco, F., D’Amico, R. et al. Facioscapulohumeral muscular dystrophy: new insights from compound heterozygotes and implication for prenatal genetic counselling. J. Med. Genet. (2011).

9 Lemmers, R. J. L. F., van der Wielen, M., Bakker, E. \& van der Maarel, S. Molecular diagnosis of FSHD. in FSHD Facioscapulohumeral Muscular Dystrophy: Clinical Medicine and Molecular Cell Biology. Upadhyaya, M. \& Cooper, D. N. (eds) 211-234 (BIOS Scientific Publishers, New York, NY, 2004).

10 Goto, K., Nishino, I. \& Hayashi, Y. K. Rapid and accurate diagnosis of facioscapulohumeral muscular dystrophy. Neuromuscul. Disord. 16, 256-261 (2006).

11 Nguyen, K., Walrafen, P., Bernard, R., Attarian, S., Chaix, C., Vovan, C. et al. Molecular combing reveals allelic combinations in facioscapulohumeral dystrophy. Ann. Neurol. 70, 627-633 (2011).

12 Fitzsimons, R. B. Retinal vascular disease and the pathogenesis of facioscapulohumeral muscular dystrophy. A signalling message from Wnt? Neuromuscul. Disord. 21, 263-271 (2011).

13 Goto, K., Lee, J. H., Matsuda, C., Hirabayashi, K., Kojo, T., Nakamura, A. et al. DNA rearrangements in Japanese facioscapulohumeral muscular dystrophy patients: clinical correlations. Neuromuscul. Disord. 5, 201-208 (1995).

14 Dorobek, M. \& Kabzinska, D. A severe case of facioscapulohumeral muscular dystrophy (FSHD) with some uncommon clinical features and a short $4 \mathrm{q} 35$ fragment. Eur. J. Paediatr. Neurol. 8, 313-316 (2004).

15 Tsuji, M., Kinoshita, M., Imai, Y., Kawamoto, M. \& Kohara, N. Facioscapulohumeral muscular dystrophy presenting with hypertrophic cardiomyopathy: a case study. Neuromuscul. Disord. 19, 140-142 (2009).

16 Wohlgemuth, M., van der Kooi, E. L., van Kesteren, R. G., van der Maarel, S. M. \& Padberg, G. W. Ventilatory support in facioscapulohumeral muscular dystrophy. Neurology 63, 176-178 (2004).

17 Carter, G. T. \& Bird, T. D. Ventilatory support in facioscapulohumeral muscular dystrophy. Neurology 64, 401 (2005).

18 Saito, A., Higuchi, I., Nakagawa, M., Saito, M., Uchida, Y., Inose, M. et al. An overexpression of fibroblast growth factor (FGF) and FGF receptor 4 in a severe clinical phenotype of facioscapulohumeral muscular dystrophy. Muscle Nerve 23, 490-497 (2000).

19 Balatsouras, D. G., Korres, S., Manta, P., Panousopoulou, A. \& Vassilopoulos, D. Cochlear function in facioscapulohumeral muscular dystrophy. Otol. Neurotol. 28, 7-10 (2007).

20 Matsuzaka, T., Sakuragawa, N., Terasawa, K. \& Kuwabara, H. Facioscapulohumeral dystrophy associated with mental retardation, hearing loss, and tortuosity of retinal arterioles. J. Child Neurol. 1, 218-223 (1986).

21 Ehrlich, M. Exploring hypotheses about the molecular etiology of FSHD: loss of heterochromatin spreading and other long-range interaction models. in FSHD
Facioscapulohumeral Muscular Dystrophy: Molecular Cell Biology \& Clinical Medicine. Cooper, D. N. \& Upadhyaya, M. (eds) 253-276 (BIOS Scientific Pub, New York, NY, 2004).

22 van der Maarel, S. M., Frants, R. R. \& Padberg, G. W. Facioscapulohumeral muscular dystrophy. Biochim. Biophys. Acta 1772, 186-194 (2007).

23 Alexiadis, V., Ballestas, M. E., Sanchez, C., Winokur, S., Vedanarayanan, V., Warren, M. et al. RNAPol-ChIP analysis of transcription from FSHD-linked tandem repeats and satellite DNA. Biochem. Biophys. Acta (1769)29-40, 2007.

24 Gabellini, D., Green, M. R. \& Tupler, R. Inappropriate gene activation in FSHD: a repressor complex binds a chromosomal repeat deleted in dystrophic muscle. Cell 110, 339-348 (2002).

25 Jiang, G., Yang, F., van Overveld, P. G., Vedanarayanan, V., van der Maarel, S. \& Ehrlich, M. Testing the position-effect variegation hypothesis for facioscapulohumeral muscular dystrophy by analysis of histone modification and gene expression in subtelomeric 4q. Hum. Mol. Genet. 12, 2909-2921 (2003).

26 Gabriels, J., Beckers, M. C., Ding, H., De Vriese, A., Plaisance, S., van der Maarel, S. M. et al. Nucleotide sequence of the partially deleted D4Z4 locus in a patient with FSHD identifies a putative gene within each $3.3 \mathrm{~kb}$ element. Gene 236, 25-32 (1999).

27 Dixit, M., Ansseau, E., Tassin, A., Winokur, S., Shi, R., Qian, H. et al. DUX4, a candidate gene of facioscapulohumeral muscular dystrophy, encodes a transcriptional activator of PITX1. Proc. Natl Acad. Sci. USA 104, 18157-18162 (2007).

28 Bosnakovski, D., Lamb, S., Simsek, T., Xu, Z., Belayew, A., Perlingeiro, R. et al. DUX4c, an FSHD candidate gene, interferes with myogenic regulators and abolishes myoblast differentiation. Exp. Neurol. 214, 87-96 (2008).

29 Wuebbles, R. D., Hanel, M. L. \& Jones, P. L. F. S. H. D. region gene 1 (FRG1) is crucial for angiogenesis linking FRG1 to facioscapulohumeral muscular dystrophyassociated vasculopathy. Dis. Model Mech. 2, 267-274 (2009).

30 Klooster, R., Straasheijm, K., Shah, B., Sowden, J., Frants, R., Thornton, C. et al. Comprehensive expression analysis of FSHD candidate genes at the mRNA and protein level. Eur. J Hum. Genet. 17, 1615-1624 (2009).

31 Winokur, S. T., Chen, Y. W., Masny, P. S., Martin, J. H., Ehmsen, J. T., Tapscott, S. J. et al. Expression profiling of FSHD muscle supports a defect in specific stages of myogenic differentiation. Hum. Mol. Genet. 12, 2895-2907 (2003).

32 Osborne, R. J., Welle, S., Venance, S. L., Thornton, C. A. \& Tawil, R. Expression profile of FSHD supports a link between retinal vasculopathy and muscular dystrophy. Neurology 68, 569-577 (2007).

33 Lemmers, R. J., Wohlgemuth, M., van der Gaag, K. J., van der Vliet, P. J., van Teijlingen, C. M., de Knijff, P. et al. Specific sequence variations within the $4 \mathrm{q} 35$ region are associated with facioscapulohumeral muscular dystrophy. Am. J. Hum. Genet. 81, 884-894 (2007)

34 Tsumagari, K., Chen, D., Hackman, J. R., Bossler, A. D. \& Ehrlich, M. FSH dystrophy and a subtelomeric $4 \mathrm{q}$ haplotype: a new assay and associations with disease. J Med. Genet. 47, 745-751 (2010).

35 Spurlock, G., Jim, H. P. \& Upadhyaya, M. Confirmation that the specific SSLP microsatellite allele 4qA161 segregates with fascioscapulohumeral muscular dystrophy (FSHD) in a cohort of multiplex and simplex FSHD families. Muscle Nerve 42, 820-821 (2010).

36 Leidenroth, A. \& Hewitt, J. E. A family history of DUX4: phylogenetic analysis of DUXA, B, C and Duxbl reveals the ancestral DUX gene. BMC Evol. Biol. 10, 364 (2010)

37 Tupler, R., Berardinelli, A., Barbierato, L., Frants, R., Hewitt, J. E., Lanzi, G. et al. Monosomy of distal $4 \mathrm{q}$ does not cause facioscapulohumeral muscular dystrophy. J. Med. Genet. 33, 366-370 (1996).

38 Xu, X., Tsumagari, K., Sowden, J., Tawil, R., Boyle, A. P., Song, L. et al. DNasel hypersensitivity at gene-poor, FSH dystrophy-linked 4q35.2. Nucleic Acids Res. 37, 7381-7393 (2009).

39 de Greef, J. C., Lemmers, R. J., van Engelen, B. G., Sacconi, S., Venance, S. L., Frants, R. R. et al. Common epigenetic changes of D4Z4 in contraction-dependent and contraction-independent FSHD. Hum. Mutat. 30, 1-11 (2009).

40 Yamanaka, G., Goto, K., Ishihara, T., Oya, Y., Miyajima, T., Hoshika, A. et al. FSHDlike patients without 4q35 deletion. J Neurol. Sci. 219, 89-93 (2004).

41 Leidenroth, A., Sorte, H. S., Gilfillan, G., Ehrlich, M., Lyle, R. \& Hewitt, J. E. Diagnosis by sequencing: correction of misdiagnosis from FSHD2 to LGMD2A by whole-exome analysis. Eur. J Hum. Genet. 20, 999-1003 (2012).

42 Snider, L., Asawachaicharn, A., Tyler, A. E., Geng, L. N., Petek, L. M., Maves, L. et al. RNA transcripts, miRNA-sized fragments and proteins produced from D4Z4 units: new candidates for the pathophysiology of facioscapulohumeral dystrophy. Hum. Mol. Genet. 18, 2414-2430 (2009).

43 Mattick, J. S. \& Makunin, I. V. Non-coding RNA. Hum. Mol. Genet. 15 (Spec No 1), R17-R29 (2006)

44 Mosch, K., Franz, H., Soeroes, S., Singh, P. B. \& Fischle, W. HP1 recruits activity-dependent neuroprotective protein to $\mathrm{H} 3 \mathrm{~K} 9 \mathrm{me} 3$ marked pericentromeric heterochromatin for silencing of major satellite repeats. PLoS One 6, e15894 (2011).

45 Szpakowski, S., Sun, X., Lage, J. M., Dyer, A., Rubinstein, J., Kowalski, D. et al. Loss of epigenetic silencing in tumors preferentially affects primate-specific retroelements. Gene 448, 151-167 (2009).

46 Zeng, W., de Greef, J. C., Chen, Y. Y., Chien, R., Kong, X., Gregson, H. C. et al. Specific loss of histone $\mathrm{H} 3$ lysine 9 trimethylation and HP1gamma/cohesin binding at D4Z4 repeats is associated with facioscapulohumeral dystrophy (FSHD). PLoS Genet. 5, e1000559 (2009). 
47 Tsien, F., Sun, B., Hopkins, N. E., Vedanarayanan, V., Figlewicz, D., Winokur, S. et al. Hypermethylation of the FSHD syndrome-linked subtelomeric repeat in normal and FSHD cells but not in ICF syndrome cells. Molec. Gen. Metab. 74, 322-331 (2001).

48 de Greef, J. C., Wohlgemuth, M., Chan, O. A., Hansson, K. B., Smeets, D., Frants R. R. et al. Hypomethylation is restricted to the D4Z4 repeat array in phenotypic FSHD. Neurology 69, 1018-1026 (2007)

49 Lyle, R., Wright, T. J., Clark, L. N. \& Hewitt, J. E. The FSHD-associated repeat, D4Z4, is a member of a dispersed family of homeobox-containing repeats, subsets of which are clustered on the short arms of the acrocentric chromosomes. Genomics 28, 389-397 (1995).

50 Winokur, S. T., Bengtsson, U., Vargas, J. C., Wasmuth, J. J., Altherr, M. R., Weiffenbach, B. et al. The evolutionary distribution and structural organization of the homeobox-containing repeat D4Z4 indicates a functional role for the ancestral copy in the FSHD region. Hum. Mol. Genet. 5, 1567-1575 (1996).

51 Foronda, D., de Navas, L. F., Garaulet, D. L. \& Sanchez-Herrero, E. Function and specificity of Hox genes. Int. J. Dev. Biol. 53, 1404-1419 (2009).

52 Richards, M., Coppee, F., Thomas, N., Belayew, A. \& Upadhyaya, M. Facioscapulohumeral muscular dystrophy (FSHD): an enigma unravelled? Hum. Genet. 131, 325-340 (2011).

53 Tsumagari, K., Chang, S. -C., Lacey, M., Baribault, C., Chittur, S. V., Sowden, J. et al. Gene expression during normal and FSHD myogenesis. BMC Med. Genomics 4, 67 (2011).

54 Wood-Allum, C., Brennan, P., Hewitt, M., Lowe, J., Tyfield, L. \& Wills, A. Clinical and histopathological heterogeneity in patients with 4q35 facioscapulohumeral muscula dystrophy (FSHD). Neuropathol. Appl. Neurobiol. 30, 188-191 (2004).

55 Reed, P., Porter, N. C., Strong, J., Pumplin, D. W., Corse, A. M., Luther, P. W. et al. Sarcolemmal reorganization in facioscapulohumeral muscular dystrophy. Ann. Neurol. 59, 289-297 (2006).

56 Carosio, S., Berardinelli, M. G., Aucello, M. \& Musaro, A. Impact of ageing on muscle cell regeneration. Ageing Res. Rev. 10, 35-42 (2011).

57 Charge, S. B. \& Rudnicki, M. A. Cellular and molecular regulation of muscle regeneration. Physiol. Rev. 84, 209-238 (2004).

58 Scharner, J. \& Zammit, P. S. The muscle satellite cell at 50: the formative years. Skelet. Muscle 1, 28 (2011).

59 Bosnakovski, D., Xu, Z., Gang, E. J., Galindo, C. L., Liu, M., Simsek, T. et al. An isogenetic myoblast expression screen identifies DUX4-mediated FSHD-associated molecular pathologies. EMBO J. 27, 2766-2779 (2008).

60 Homma, S., Chen, J. C., Rahimov, F., Beermann, M. L., Hanger, K., Bibat, G. M. et al. A unique library of myogenic cells from facioscapulohumeral muscular dystrophy subjects and unaffected relatives: family, disease and cell function. Eur. J. Hum. Genet. 20, 204-210 (2011).

61 Geng, L. N., Yao, Z., Snider, L., Fong, A. P., Cech, J. N., Young, J. M. et al. DUX4 activates germline genes, retroelements, and immune mediators: Implications for facioscapulohumeral dystrophy. Dev. Cell 22, 38-51 (2012).

62 Wallace, L. M., Garwick, S. E., Mei, W., Belayew, A., Coppee, F., Ladner, K. J. et al. DUX4, a candidate gene for facioscapulohumeral muscular dystrophy, causes p53dependent myopathy in vivo. Ann. Neurol. 69, 540-552 (2011).

63 Vanderplanck, C., Ansseau, E., Charron, S., Stricwant, N., Tassin, A., LaoudjChenivesse, D. et al. The FSHD atrophic myotube phenotype is caused by DUX4 expression. PLoS One 6, e26820 (2011).

64 Carter, M. G., Stagg, C. A., Falco, G., Yoshikawa, T., Bassey, U. C., Aiba, K. et al. An in situ hybridization-based screen for heterogeneously expressed genes in mouse ES cells. Gene. Expr. Patterns 8, 181-198 (2008).

65 Kobayashi, T. \& Kageyama, R. Hes1 regulates embryonic stem cell differentiation by suppressing Notch signaling. Genes Cells 15, 689-698 (2010).

66 Suzuki, N., Furusawa, C. \& Kaneko, K. Oscillatory protein expression dynamics endows stem cells with robust differentiation potential. PLoS One 6, e27232 (2011)

67 Falco, G., Lee, S. L., Stanghellini, I., Bassey, U. C., Hamatani, T. \& Ko, M. S. Zscan4 a novel gene expressed exclusively in late 2-cell embryos and embryonic stem cells. Dev. Biol. 307, 539-550 (2007)

68 Tanaka, T. S. Transcriptional heterogeneity in mouse embryonic stem cells. Reprod Fertil. Dev. 21, 67-75 (2009).

69 Tupler, R., Perini, G., Pellegrino, M. A. \& Green, M. R. Profound misregulation of muscle-specific gene expression in facioscapulohumeral muscular dystrophy. Proc Natl Acad. Sci. USA 96, 12650-12654 (1999).

70 Celegato, B., Capitanio, D., Pescatori, M., Romualdi, C., Pacchioni, B., Cagnin, S. et al. Parallel protein and transcript profiles of FSHD patient muscles correlate to the D4Z4 arrangement and reveal a common impairment of slow to fast fibre differentiation and a general deregulation of MyoD-dependent genes. Proteomics 6, 53035321 (2006)

71 Arashiro, P., Eisenberg, I., Kho, A. T., Cerqueira, A. M., Canovas, M., Silva, H. C. et al. Transcriptional regulation differs in affected facioscapulohumeral muscular dystrophy patients compared to asymptomatic related carriers. Proc. Natl Acad. Sci. USA 106, 6220-6225 (2009).

72 Cheli, S., Francois, S., Bodega, B., Ferrari, F., Tenedini, E., Roncaglia, E. et al. Expression profiling of FSHD-1 and FSHD-2 cells during myogenic differentiation evidences common and distinctive gene dysregulation patterns. PLoS One 6, e20966 (2011).

73 Fitzsimons, R. B. Facioscapulohumeral dystrophy: the role of inflammation. Lancet 344, 902-903 (1994)

74 Arahata, K., Ishihara, T., Fukunaga, H., Orimo, S., Lee, J. H., Goto, K. et al. Inflammatory response in facioscapulohumeral muscular dystrophy (FSHD): immunocytochemical and genetic analyses. Muscle Nerve 2, S56-S66 (1995).

75 Kan, H. E., Scheenen, T. W., Wohlgemuth, M., Klomp, D. W., van Loosbroek Wagenmans, I., Padberg, G. W. et al. Quantitative MR imaging of individual muscle involvement in facioscapulohumeral muscular dystrophy. Neuromuscul. Disord. 19, 357-362 (2009).

76 Frisullo, G., Frusciante, R., Nociti, V., Tasca, G., Renna, R., Iorio, R. et al. CD8( + ) T Cells infacioscapulohumeral muscular dystrophy patients with inflammatory features at muscle MRI. J. Clin. Immunol. 31, 155-166 (2011).

77 Sun, G., Haginoya, K., Wu, Y., Chiba, Y., Nakanishi, T., Onuma, A. et al. Connective tissue growth factor is overexpressed in muscles of human muscular dystrophy. J. Neurol. Sci. 267, 48-56 (2008).

78 Laoudj-Chenivesse, D., Carnac, G., Bisbal, C., Hugon, G., Bouillot, S., Desnuelle, C. et al. Increased levels of adenine nucleotide translocator 1 protein and response to oxidative stress are early events in facioscapulohumeral muscular dystrophy muscle. J. Mol. Med. 83, 216-224 (2005).

79 Hirata, T., Amano, T., Nakatake, Y., Amano, M., Piao, Y., Hoang, H. G. et al. Zscan4 transiently reactivates early embryonic genes during the generation of induced pluripotent stem cells. Sci. Rep. 2, 208 (2012).

80 Hryniuk, A., Grainger, S., Savory, J. G. \& Lohnes, D. Cdx function is required for maintenance of intestinal identity in the adult. Dev. Biol. 363, 426-437 (2012).

81 Gundersen, K. Excitation-transcription coupling in skeletal muscle: the molecular pathways of exercise. Biol. Rev. Camb. Philos. Soc. 86, 564-600 (2011).

82 Barber, B. A. \& Rastegar, M. Epigenetic control of Hox genes during neurogenesis, development, and disease. Ann. Anat. 192, 261-274 (2010).

83 Soshnikova, N. \& Duboule, D. Epigenetic regulation of vertebrate Hox genes: a dynamic equilibrium. Epigenetics 4, 537-540 (2009).

84 MacLean, 2nd J. A. \& Wilkinson, M. F. The Rhox genes. Reproduction 140, 195-213 (2010)

$85 \mathrm{Li}$, J., Dani, J. A. \& Le, W. The role of transcription factor Pitx3 in dopamine neuron development and Parkinson's disease Curr. Top Med. Chem. 9, 855-859 (2009).

86 Lepper, C., Conway, S. J. \& Fan, C. M. Adult satellite cells and embryonic muscle progenitors have distinct genetic requirements. Nature 460, 627-631 (2009).

87 Tschopp, P. \& Duboule, D. A genetic approach to the transcriptional regulation of Hox gene clusters. Annu. Rev. Genet. 45, 145-166 (2011).

88 Boutet, S. C., Biressi, S., Iori, K., Natu, V. \& Rando, T. A. Taf1 regulates Pax3 protein by monoubiquitination in skeletal muscle progenitors. Mol. Cell 40, 749-761 (2010).

89 Chen, J. F., Tao, Y., Li, J., Deng, Z., Yan, Z., Xiao, X. et al. microRNA-1 and microRNA 206 regulate skeletal muscle satellite cell proliferation and differentiation by repressing Pax7. J. Cell. Biol. 190, 867-879 (2010).

90 Liu, D. X. \& Lobie, P. E. Transcriptional activation of p53 by Pitx1. Cell Death Differ 14, 1893-1907 (2007).

91 Daftary, G. S. \& Taylor, H. S. Endocrine regulation of HOX genes. Endocr. Rev. 27, 331-355 (2006).

92 Mann, R. S., Lelli, K. M. \& Joshi, R. Hox specificity unique roles for cofactors and collaborators. Curr. Top Dev. Biol. 88, 63-101 (2009).

93 Mallo, M., Wellik, D. M. \& Deschamps, J. Hox genes and regional patterning of the vertebrate body plan. Dev. Biol. 344, 7-15 (2010).

94 Clapp, J., Mitchell, L. M., Bolland, D. J., Fantes, J., Corcoran, A. E., Scotting, P. J. et al. Evolutionary conservation of a coding function for D4Z4, the tandem DNA repeat mutated in facioscapulohumeral muscular dystrophy. Am. J. Hum. Genet. 81, 264-279 (2007).

95 Wu, S. L., Tsai, M. S., Wong, S. H., Hsieh-Li, H. M., Tsai, T. S., Chang, W. T. et al. Characterization of genomic structures and expression profiles of three tandem repeats of a mouse double homeobox gene: Duxbl. Dev. Dyn. 239, 927-940 (2010)

96 Kawazu, M. Yamamoto, G., Yoshimi, M. Yamamoto, K. Asai, T., Ichikawa, M. et al. Expression profiling of immature thymocytes revealed a novel homeobox gene that regulates double-negative thymocyte development. J. Immunol. 179, 5335-5345 (2007).

97 Barro, M., Carnac, G., Flavier, S., Mercier, J., Vassetzky, Y \& Laoudj-Chenivesse, D. Myoblasts from affected and non affected FSHD muscles exhibit morphological differentiation defects. J. Cell Mol. Med. 275-289 (2010).

98 Baumer, N., Sandstede, M. L., Diederichs, S., Kohler, G. Readhead, C., Ji, P. et al. Analysis of the genetic interactions between Cyclin A1, Atm and p53 during spermatogenesis. Asian J. Androl. 9, 739-750 (2007).

99 Bentzinger, C. F., von Maltzahn, J. \& Rudnicki, M. A. Extrinsic regulation of satellite cell specification. Stem Cell Res. Ther. 1, 27 (2011).

100 Kirkpatrick, L. J., Yablonka-Reuveni, Z. \& Rosser, B. W. Retention of Pax3 expression in satellite cells of muscle spindles. J. Histochem. Cytochem. 58, 317-327 (2010). 